\title{
Analytical Modelling of Structure-Borne Sound Transmission through I-junction using Chebyshev-Ritz method on Cascaded Rectangular Plate-Cavity System
}

\author{
Cheng Siong Chin", Ji Xi \\ ${ }^{\text {a }}$ Faculty of Science, Agriculture and Engineering, \\ Newcastle University in Singapore \\ Singapore 599493
}

\begin{abstract}
A Chebyshev-Ritz based analytical model is proposed to investigate I-junction within the structuralacoustic model of a cascaded rectangular plate-cavity system. By considering of the structural interconnection force and the moment at edges and structural-acoustic interaction on the interface, the structural and acoustic systems are coupled. Two-dimensional and three-dimensional Chebyshev Polynomial series are used to present the unknown panel displacements and the sound pressure field variable inside the cavities, respectively. The effectiveness and correctness of the proposed model on an I-junction in a typical marine offshore platform are verified with those calculated from Finite Element Analysis. The influence of boundary conditions, structural coupling, plate properties, and size of the source-to-receiver cavities on the offshore platform on structure-borne sound transmission are analyzed and addressed. Numerical examples are simulated for several different configurations. It is shown that the boundary conditions, structural coupling manner, plate properties, and the volume ratio of the source-to-receiver cavity will change the structure-borne sound transmission characteristics of the cascaded rectangular plate-cavity system. With the proposed approach, a better prediction can be obtained for structure-borne sound transmission via proper tuning of the cascaded rectangular platecavity system on the offshore platform.
\end{abstract}

Keywords: structure-borne sound transmission; cascaded rectangular plate-cavity system; RayleighRitz; Chebyshev polynomial; offshore platform; finite element analysis

*Corresponding author. cheng.chin@ncl.ac.uk (Cheng Siong Chin), Tel: 6569086013 


\section{Introduction}

Accurate structure-borne sound transmission prediction is desirable for marine and offshore installations that are characterized by thin-plate steel construction and clustering of many compartments or rooms. The low mass and high stiffness construction result in a low sound transmission loss over the large frequency band. Apart from the direct transmission between rooms through a single component (i.e., single panel), the global structural-borne noise transmission affects several compartments in the marine and offshore platform. There are three parts involved in the structure-borne noise transmission between compartments, namely: i) structural loading by external dynamic loading such as dynamic mechanical force, sound wave impingement; ii) structural response and iii) interior acoustic response. The structure vibration induced by the external loading is propagated in the form of longitudinal, torsional, and transverse waves. These wave types are coupled and converted along the transmission path. For example, a pure transverse wave impinges on a junction, and other wave types are induced. However, at the receiving end of the transmission line, only the transverse waves are involved due to other wave are weakly coupled to the cavity. The structure-borne transmission includes the vibroacoustic behaviors of all coupled structure and cavity components along the transmission path.

Existing studies on the vibro-acoustic behavior of complex configuration are based on the deterministic analysis such as the finite element method (FEM) [1]. These space discretization based methods can be used to model the response of complex structures. However, in the frequency range for structure-borne sound studies is up to $8000 \mathrm{~Hz}$ and the number of elements required is quite large to achieve satisfactory performance. The energy-based method such as statistical energy analysis (SEA) [2-3] for structureborne sound transmission at high frequencies can be used. However, Craik et al. [4] reported that it was difficult to describe the coupling between the structure and cavity, making sound transmission loss calculation unreliable. Analytical methods using an analytical model can be tuned without changing the solution procedure. In addition, the analytical approach does not restrict the frequency as compared to the energy and discretization method as seen above. The dynamic characteristics of a cavity subjected to individual vibrating panels have been extensively studied [5-17]. The structure-borne sound transmission is usually solved semi-analytically using modal coupling approaches [13] [6, 17-20] that requires prior modal information.

The Rayleigh method [21] works by the principle that the energy of a vibrating system interchange between the potential and kinetic form without dissipation at each natural mode. By using a set of admissible trial function for the mode shapes and assuming simple harmonic motion, the equalization of maximum potential energy and the maximum kinetic energy yields the dynamic quantities. Ritz [22] generalized the Rayleigh method by assuming a set of admissible trial functions, each having independent amplitude coefficients. The approximations for the frequency can be achieved by minimizing the energy function with respect to each coefficient. Ritz demonstrated this method on a square plate under free boundary condition with no exact solution. However, the accuracy and effectiveness of using the Rayleigh-Ritz technique for each unknown Chebyshev polynomial series coefficients of the structural-acoustic coupling model could be improved.

The Chebyshev polynomials of the first kind as the admissible function for structure dynamic has become popular due to the excellent function approximation for Fourier series[23], rapid convergence, and better stability in numerical operation. It was applied to solving vibration problems with uniform boundary conditions such as plain rectangular plates [24], stepped beam [25], annular sector plates [26], laminate beams [27], rectangular plates with cutout [28]. The same approach of using Chebyshev polynomials was also used for solving vibro-acoustic problem on a single three-dimensional enclosure such as annular segment cavity [29], cylindrical shell [30], typical rail and aerospace structures [31], 
regular rectangular cavity with arbitrary impedance walls [32] and elastically restrained panel backed by irregular sound space [6-8,12-14, 16-17, 33-34]. Recently, an extension to non-uniform boundary conditions [35] was proposed on single laminated rectangular plate-cavity coupling system. Subsequently, another recent paper [36] worked on the vibro-acoustic coupling model of a single elastically restrained plate backed by the inclined wall was proposed. The results showed that the inclination arrangement could modify the panel-cavity structural-acoustic coupling pattern. In addition, the structural-acoustic coupling model [37] of a single annular segment thin plate with various elastic boundary constraints and different impedance-wall boundary conditions was shown using the same Rayleigh-Ritz energy technique. With further interest, a coupled acoustic system comprising a partially opened cavity [38] coupled with a flexible plate using the Rayleigh-Ritz procedure based on the energy expressions for the coupled system was performed.

However, most of the literature examined on single or standalone structural-acoustic model of a platecavity system problem without exploring when there exists more than one rectangular plate-cavity systems forming a cascaded plate-cavity system. The underlying plate-cavity system connectivity can be represented by an I-junction (situated between the two rectangular plate-cavity systems) where there is more than one room on the offshore platform. Furthermore, the impacts to different boundary spring stiffness, structural, acoustic and dimensional properties on the structure-borne sound transmission for the I-junction within the structural-acoustic model of a cascaded rectangular plate-cavity system are not examined? Hence, there exists an apparent gap in the current literature. Motivated by such limitation in literature, an analytical model for the structure-borne sound transmission path of the structuralacoustic model of a cascaded rectangular plate-cavity system is therefore required.

In this paper, the structural-acoustic model of a cascaded rectangular plate-cavity system under general boundary condition is proposed in a unified energy formulation. Instead of repeating the theory found in most literature, more emphasis on the aspect of the final equations of motion that is unique to this paper is described. The simplified configuration is set up that consists of two cavities separated by five coupled plates. The problem is solved in the frequency domain. The Rayleigh-Ritz approach serves as the theoretical framework to develop the vibro-acoustic interaction model. To improve the convergence rate, the Chebyshev-Lagrangian method is employed to formulate the structural and acoustic energy functions. According to the principal of compatibility and consistency, the displacement components of the thin plate and the sound pressure inside the acoustic domain are approximated by the double and triple Chebyshev orthogonal polynomials, respectively. To provide insight into the physical phenomena that dominate the structure-borne sound transmission through I-junction within the structural-acoustic model of the cascaded rectangular plate-cavity system, the proposed method is validated by checking the dynamic responses with those obtained from finite element software. Lastly, several parametric studies are carried out on the proposed model by analyzing the boundary conditions, structural coupling manner, plate properties, and the volume ratio of the source-to-receiver cavity on the structure-borne transmission characteristics in the cavities. It enables the cascaded rectangular plate-cavity system model to handle wide frequency range by choosing sufficient truncated terms for the plate displacements and sound pressure functions.

The contributions are summarized as follows. First, the structure-borne sound transmission via the Ijunction of a cascaded rectangular plate-cavity system under general boundary condition is modeled using a Chebyshev-Ritz method. The coupling forces and moments between plates and cavity are used in the computation. Second, the influences of several parameters on the structure-borne sound transmission within the configuration are investigated. The outcome of these investigations enables understanding of the structure-borne sound transmission mechanisms within the cascaded rectangular 
plate-cavity system to guide engineering noise control on offshore platform compartments or any compartments on land.

\section{Theoretical Formulation}

\subsection{Model description of Cascaded Rectangular Plate-Cavity System}

The goal is to formulate an analytical model for the vibroacoustic problem of quite large dimensions in a wide frequency range, with a reduction in computational costs. By considering structure-borne sound transmission rather than merely sound transmission through a single wall, a simplified geometry can be proposed. The proposed analytical model of a cascaded rectangular plate-cavity system with five coupled plates elastically restrained and coupled along boundary edges and two cavities with rigid walls can be seen in Figure 1. Note that the I-junction is situated between the two rectangular plate-cavity systems. The two cavities that are semi-enclosed with three homogeneous and isotropic flat surface are denoted as cavity $a$ and $b$, respectively. Flat plates are numbered (P1, P2, P3, P4 and P5) and plate dimensions are denoted as $L_{i, x}, L_{i, y}, L_{i, z}$, respectively in the Cartesian coordinates. The built-up system with a superposed reference between the global coordinate and the local coordinate of the common plate is aimed to illustrate the dynamics of acoustic and structure domain.

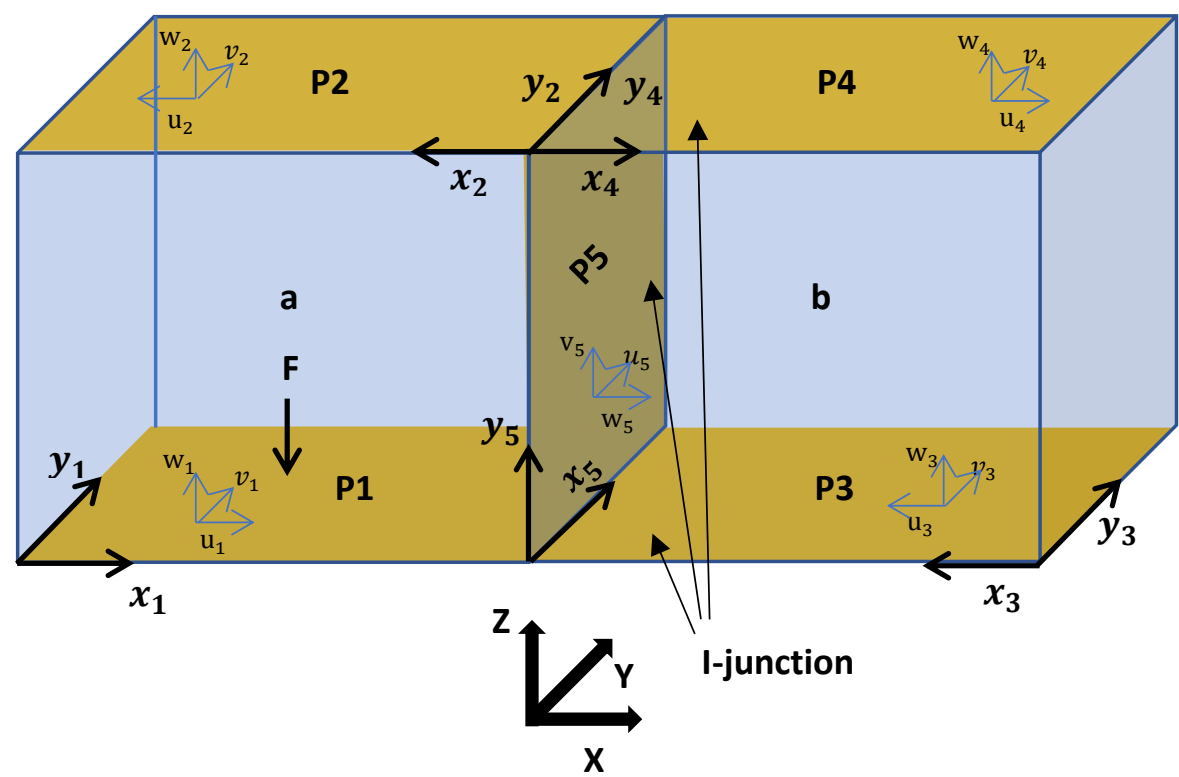

Figure 1 Schematic diagram of structural-acoustic model of a cascaded rectangular plate-cavity system separated by I-junction on offshore platform

On the structure side, the vibration transmits in the form of boundary forces and moments at the coupling edges. The dynamic behavior of these coupled plates is quite different due to the connection types and boundary conditions. Applying uniformly distributed artificial spring to restrain the edges elastically for the structural coupling, can efficiently model the classical boundary conditions such as simply supported, clamped, free and guided conditions. The general boundary condition for the flexural and in-plane vibrations of rectangular plates can be specified using four types of springs distributed along each edge. They are three translational springs and one rotational spring. These springs are distributed uniformly along and around the $X, Y$ and $Z$ axis to simulate the given boundary conditions. 
Four additional groups of springs are uniformly arranged at the coupling edges to represent general connection manners between the contiguous plates. All spring constants are depicted in such a way that indicates the type and the location. The parameters $K_{w}, k_{w}, k_{p}$, and $k_{n}$ refer to the rotational spring stiffness, translational spring stiffness, the in-plane linear spring stiffness to the edge, and in the normal direction to the edge, respectively. The other parameters $K_{c}, k_{c w}, k_{c u}$, and $k_{c v}$ are the coupling stiffness of the artificially connected adjacent plates. By the definition of geometrical boundaries, arbitrary set of classical boundary conditions at the edges of plates can be obtained by an appropriate choice of the stiffness values. The spring stiffness can be quite high for a clamped edge and zero for a free boundary condition. The force equilibrium can be achieved by the actions and reactions on the connected panels.

For both cavities, assumed the contained fluid is inviscid, and acoustic pressure directly affects the transverse deformation of the plates. At steady state, the continuity condition of structure-acoustic coupling system on the structural-acoustic interface is to match the air particle velocity with the structural transverse velocities such that

$$
\nabla p(x, y, z)=-j \rho_{0} \omega w_{i}(x, y, z)
$$

where $\nabla=\left[\begin{array}{lll}\partial / \partial x & \partial / \partial y & \partial / \partial z\end{array}\right]^{\mathrm{T}}$ represents the gradient operator, $p(x, y, z)$ is the acoustic pressure in the rectangular cavity, $j=\sqrt{-1} ; \rho_{0}$ is the fluid density, $\omega$ denotes the angular frequency of the wave and $w_{i}(x, y, z)$ is the transverse displacement of the plate $i$.

\subsection{Energy function of the coupled structural domain and acoustic domain}

The linear structural model of the structural-acoustic system based on Kirchhoff's thin plate theory is established by decomposing the structural energy into subsets corresponding to the exterior surfaces and interior surfaces that yield the general form of functions.

$$
L^{S}=\sum_{i=1}^{5}\left(V_{i}^{S}-T_{i}^{S}\right)+\sum_{i, i+1}^{6} V_{i, i+1}^{S S}+\sum_{i_{e x}=1}^{4} W_{i_{e x}}^{A S}+W_{i_{i n}}^{A S}-W_{i}^{F}
$$

where $V_{i}^{S}$ is the potential energy done due to transverse and in-plane deformation, $T_{i}^{S}$ is the kinetic energy of $i$ th plate. $W_{i}^{F}$ is the work done by external loads, $W_{i_{i n}}^{A S}$ denotes the additional energy by the resulted acoustic pressure loading from the cavity $a$ and $b$ simultaneously. $W_{i_{e x}}^{A S}$ is the work done by acoustic loading to the exterior surfaces. $V_{i, i+1}^{S S}$ represents the potential energy due to the structural coupling between the plate $i$ and its adjacent plate $i+1$. Although the inplane deformation has little contribution to the noise radiation into the cavity, it plays a significant role in the vibration energy transmission among the connected plates. The in-plane vibration can be excited when there is a load within or parallel to the plate. Therefore, the detailed energy terms of $i$ th plate involving both transverse and in-plane motion are expressed as 


$$
\begin{aligned}
& V_{i}^{S}=\frac{D_{i}}{2} \int_{0}^{L_{i, x}} \int_{0}^{L_{i, y}}\left[\left(\frac{\partial^{2} w_{i}}{\partial x^{2}}\right)^{2}+\left(\frac{\partial^{2} w_{i}}{\partial y^{2}}\right)^{2}+2 v_{i} \frac{\partial^{2} w_{i}}{\partial x^{2}} \frac{\partial^{2} w_{i}}{\partial y^{2}}+2\left(1-v_{i}\right)\left(\frac{\partial^{2} w_{i}}{\partial x \partial y}\right)^{2}\right] d x_{i} d y_{i} \\
& +\frac{1}{2} \int_{0}^{L_{i, y}}\left[k_{w_{-} x_{i} 0} w_{i}^{2}+K_{w_{-} x_{i} 0}\left(\frac{\partial w_{i}}{\partial x}\right)^{2}\right]_{x_{i}=0} d y_{i}+\frac{1}{2} \int_{0}^{L_{i, y}}\left[k_{w_{-} x_{i}} w_{i}^{2}+K_{w_{-} x_{i}}\left(\frac{\partial w_{i}}{\partial x}\right)^{2}\right]_{x_{i}=L_{i, x}} d y_{i} \\
& +\frac{1}{2} \int_{0}^{L_{i, x}}\left[k_{w_{-} y_{i} 0} w_{i}^{2}+K_{w_{-} y_{i} 0}\left(\frac{\partial w_{i}}{\partial y}\right)^{2}\right]_{y_{i}=0} d x_{i}+\frac{1}{2} \int_{0}^{L_{i, x}}\left[k_{w_{-} y_{i}} w_{i}^{2}+K_{w_{-} y_{i} 1}\left(\frac{\partial w_{i}}{\partial y}\right)^{2}\right]_{y_{i}=L_{i, y}} d x_{i} \\
& +\frac{G_{i}}{2} \int_{0}^{L_{i, x}} \int_{0}^{L_{i, y}}\left[\left(\frac{\partial u_{i}}{\partial x_{i}}+\frac{\partial v_{i}}{\partial y_{i}}\right)^{2}-2\left(1-v_{i}\right) \frac{\partial u_{i}}{\partial x_{i}} \frac{\partial v_{i}}{\partial y_{i}}+\frac{\left(1-v_{i}\right)}{2}\left(\frac{\partial u_{i}}{\partial y_{i}}+\frac{\partial v_{i}}{\partial x_{i}}\right)^{2}\right] d x_{i} d y_{i} \\
& +\frac{1}{2} \int_{0}^{L_{i, y}}\left[k_{n_{-} x_{i}} u_{i}^{2}+k_{p_{-} x_{i}} v_{i}^{2}\right]_{x_{i}=0} d y_{i}+\frac{1}{2} \int_{0}^{L_{i, y}}\left[k_{n_{-} x_{i}} u_{i}^{2}+k_{p_{-} x_{i}} v_{i}^{2}\right]_{x_{i}=L_{i, x}} d y_{i} \\
& +\frac{1}{2} \int_{0}^{L_{i, x}}\left[k_{n_{-} y_{i}} v_{i}^{2}+k_{p_{-} y_{i}} u_{i}^{2}\right]_{y_{i}=0} d x_{i}+\frac{1}{2} \int_{0}^{L_{i, x}}\left[k_{n_{-} y_{i}} v_{i}^{2}+k_{p_{-} y_{i}} u_{i}^{2}\right]_{y_{i}=L_{i, y}} d x_{i} \\
& T_{i}^{S}=\frac{1}{2} m_{i, S} \omega_{i, S}^{2} \int_{0}^{L_{i, x}} \int_{0}^{L_{i, y}}\left(w_{i}^{2}+u_{i}^{2}+v_{i}^{2}\right) d x_{i} d y_{i} \\
& W_{i_{i n}}^{A S}=\frac{1}{2} \int_{0}^{L_{i, n}, L_{i n, y}} \int_{0}^{L_{5}} w_{5}\left(p_{i, a}-p_{i, b}\right) d x_{i_{i n}} d y_{i_{i n}} \\
& W_{i_{e x}}^{A S}=\frac{1}{2} \int_{0}^{L_{i e x, x}} \int_{0}^{L_{e x, y}, y} w_{i_{e x}} p_{i} d x_{i_{e x}} d y_{e_{e x}} \\
& W_{i}^{F}=\int_{0}^{L_{i x}} \int_{0}^{L_{i, y}} F_{i} w_{i} d x_{i} d y_{i}
\end{aligned}
$$

where $\omega_{i, S}$ is the angular frequency, $w_{i}, u_{i}$, and $v_{i}$ are the displacement components in the transverse direction and in-plane directions and $F_{i}$ is the external point force normal to the plate $i$. In addition, $L_{i, x}$ and $L_{i, y}$ are the length and height of the plate $i, D_{i}$ and $G_{i}$ are defined as $G_{i}=E h_{i} /\left(1-v^{2}\right)$ and $D_{i}=E h_{i}^{3} / 12\left(1-v^{2}\right)$. Here $E, h_{i}$, and $v$ represents Young's modulus, the thickness of the plate $i$, and Poisson's ratio, respectively.

The coupling effect of the plates is described by four groups of springs associated with the transverse moment, out-of-plane shear force, in-plane longitudinal force, and in-plane shear force. The potential energy due to plates is coupled along y-directions as shown.

$$
V_{i j}^{S S}=\frac{1}{2} \int_{0}^{L_{l}}\left[\begin{array}{l}
K_{c_{-i j}(j}\left(\left.\frac{\partial w_{i}}{\partial y_{i}}\right|_{y_{i}}-\left.\frac{\partial w_{i+1}}{\partial x_{i+1}}\right|_{x_{i+1}}\right)^{2}+k_{c w_{-}-i j}\left(\left.w_{i}\right|_{y_{i}} \pm\left. u_{i+1}\right|_{x_{i+1}}\right)^{2}+ \\
k_{c u_{-} i j}\left(\left.u_{i}\right|_{y_{i}} \pm\left. v_{i+1}\right|_{x_{i+1}}\right)^{2}+k_{c v_{-} i j}\left(\left.v_{i}\right|_{y_{i}} \pm\left. w_{i+1}\right|_{x_{i+1}}\right)^{2}
\end{array}\right] d Y
$$

The energy function for the acoustic domain $L^{A}$ is constructed by the potential energy $V^{A}$ and the kinetic energy $T^{A}$ stored in the cavity. The work contributed by the plate vibration, $W_{i_{i n}}^{A S}$ and $W_{i_{\text {ex }}}^{A S}$ can be written as

$$
L^{A}=V_{a}^{A}-T_{a}^{A}+V_{b}^{A}-T_{b}^{A}+\sum_{i_{e x}=1}^{4} W_{i_{e x}}^{A S}+W_{i_{i n}}^{A S}
$$


Assuming source strength $q$ is independent of local coordinates, the explicit expression of the potential and kinetic energies of the cavities are expressed as

$$
\begin{gathered}
V^{A}=\frac{1}{2 \rho_{0} c_{0}^{2}} \int_{0}^{L_{X}} \int_{0}^{L_{Y}} \int_{0}^{L_{z}} p^{2} d x d y d z \\
T^{A}=\frac{1}{2 \rho_{0} \omega_{A}^{2}} \int_{0}^{L_{X}} \int_{0}^{L_{y}} \int_{0}^{L_{Z}}\left[\left(\frac{\partial p}{\partial x}\right)^{2}+\left(\frac{\partial p}{\partial y}\right)^{2}+\left(\frac{\partial p}{\partial z}\right)^{2}\right] d x d y d z
\end{gathered}
$$

Considering the continuity of the displacement derivatives over the entire area of the plate, and the continuity over the structural-acoustic interface, the steady-state dynamic variables (acoustic pressure and the plate displacements) are expressed as the sum of Chebyshev polynomial of the first kind. The Chebyshev polynomial series of the first kind allow the double series and triple series. The orthogonal sets in the plate and cavity region will unify the expression for the least square method. It can provide rapid convergence and better numerical stability than other polynomial series[12]. The displacements of each plate and pressure functions are approximated by double and triplicate Chebyshev polynomial series written as follows.

$$
\begin{gathered}
w_{i}\left(\alpha_{i}, \beta_{i}\right)=\sum_{m=0}^{M} \sum_{n=0}^{N} A_{i, m, n} T_{m}\left(\alpha_{i}\right) T_{n}\left(\beta_{i}\right) \\
u_{i}\left(\alpha_{i}, \beta_{i}\right)=\sum_{m=0}^{M} \sum_{n=0}^{N} B_{i, m, n} T_{m}\left(\alpha_{i}\right) T_{n}\left(\beta_{i}\right) \\
v_{i}\left(\alpha_{i}, \beta_{i}\right)=\sum_{m=0}^{M} \sum_{n=0}^{N} C_{i, m, n} T_{m}\left(\alpha_{i}\right) T_{n}\left(\beta_{i}\right) \\
p(\alpha, \beta, \varepsilon)=\sum_{m x=0}^{M_{X}} \sum_{m y=0}^{M_{y}} \sum_{m z=0}^{M_{z}} E_{m x, m y, m z} T_{m x}(\alpha) T_{m y}(\beta) T_{m z}(\varepsilon)
\end{gathered}
$$

where $A_{i, m, n}, B_{i, m, n}, C_{i, m, n}$ and $E_{m x, m y, m z}$ are the unknown coefficients of the Chebyshev expansions to be determined. $T_{\Xi_{i}}(\chi)\left(\Xi_{i}=m_{i}, n_{i}, m x, m y, m z ; \chi=\alpha_{i}, \beta_{i}, \alpha, \beta, \varepsilon\right)$ is the one-dimensional first kind Chebyshev polynomial. Additionally, $M, N, M_{X}, M_{Y}$ and $M_{Z}$ are the total employed terms. The preferred choice of the polynomial numbers varies with the problems complexity, the geometry of the examined system and the frequency range of interest.

The Chebyshev polynomials are defined between the interval $[-1,1]$. Before substituting the displacement expansions and pressure expansions into the Lagrangian equations, mapping the real coordinate in the physical to the required range is required. Then substituting (12) to (15) into the Lagrangians equation as seen in (2) and (9) before applying the Rayleigh-Ritz procedure for each unknown Chebyshev polynomial series coefficients. The equation of motions in matrix form can be obtained.

$$
\left[\begin{array}{cc}
\mathbf{K}_{\mathrm{S}} & \mathrm{C}_{\mathrm{SA}}^{\mathrm{T}} \\
\mathbf{0} & \mathbf{K}_{\mathrm{A}}
\end{array}\right]\left[\begin{array}{l}
\boldsymbol{\Theta} \\
\boldsymbol{\Omega}
\end{array}\right]-\boldsymbol{\omega}^{2}\left[\begin{array}{cc}
\mathbf{M}_{\mathrm{S}} & \mathbf{0} \\
-\mathbf{C}_{\mathrm{SA}} & \mathbf{M}_{\mathrm{A}}
\end{array}\right]\left[\begin{array}{l}
\boldsymbol{\Theta} \\
\boldsymbol{\Omega}
\end{array}\right]=\left\{\begin{array}{l}
\mathbf{F} \\
\mathbf{0}
\end{array}\right\}
$$

where subscript $S$ and $A$ denote that the variables related to the structure and cavity, respectively. Here, $\mathbf{M}$ and $\mathbf{K}$ are the generalized global mass and stiffness matrices. The stiffness matrices of the structure 
domain $\mathbf{K}_{S}$ are formed by the transverse component $\mathbf{K}_{\mathbf{i}}^{\mathbf{T}}$, four in-plane components $\mathbf{K}_{\mathbf{i}}^{\mathbf{U}}, \mathbf{K}_{\mathbf{i}}^{\mathbf{v}}$, and structural coupling components $\mathbf{K}_{\mathbf{i}-\mathbf{i}}^{\mathrm{ss}}$ as shown.

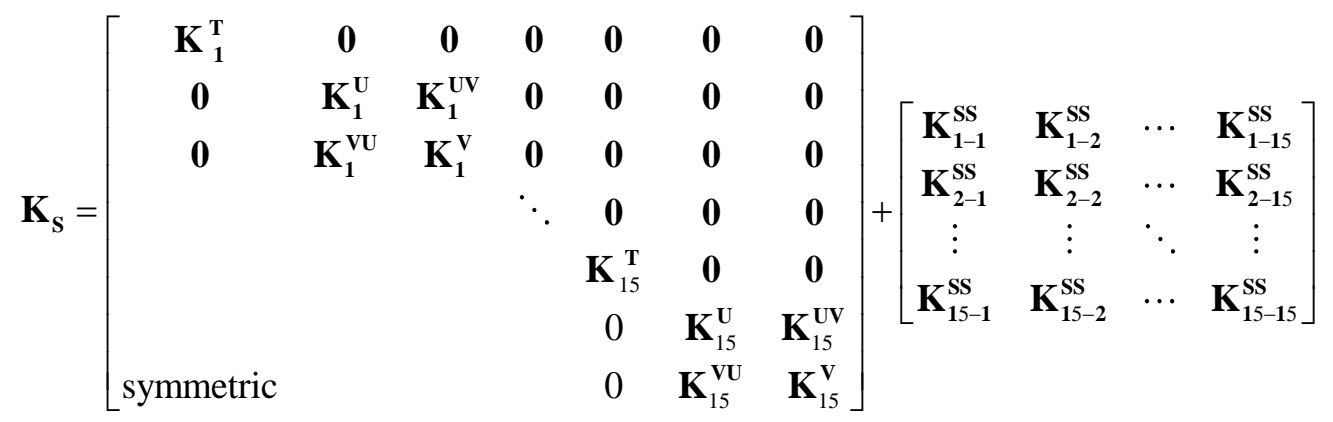

The stiffness vector of the acoustic domain $\mathbf{K}_{\mathbf{A}}$ is expressed as

$$
\mathbf{K}_{\mathrm{A}}=\left[\begin{array}{cc}
\mathbf{K}_{\mathrm{a}} & \mathbf{0} \\
\mathbf{0} & \mathbf{K}_{\mathrm{b}}
\end{array}\right]
$$

The structure mass matrix $\mathbf{M}_{\mathrm{s}}$ and acoustic mass matrix are defined as

$$
\begin{aligned}
& \mathbf{M}_{\mathrm{S}}=\operatorname{diag}\left\{\begin{array}{lllllll}
\mathbf{M}_{1} & \mathbf{M}_{1} & \mathbf{M}_{1} & \cdots & \mathbf{M}_{\mathrm{N}_{\mathrm{p}}} & \mathbf{M}_{\mathrm{N}_{\mathrm{p}}} & \mathbf{M}_{\mathrm{N}_{\mathrm{p}}}
\end{array}\right\} \\
& \mathbf{M}_{\mathbf{A}}=\operatorname{diag} \begin{cases}\mathbf{M}_{\mathbf{A}} & \left.\mathbf{M}_{\mathbf{B}}\right\}\end{cases}
\end{aligned}
$$

The structure-acoustic coupling term $\mathbf{C}_{\mathbf{S A}}$ is written as

$$
\mathbf{C}_{\mathrm{SA}}=\left[\begin{array}{ccccccccccccccc}
\mathbf{C}_{1 a}^{\mathbf{T}} & \mathbf{0} & \mathbf{0} & \mathbf{C}_{2 \mathrm{a}}^{\mathrm{T}} & \mathbf{0} & \mathbf{0} & \mathbf{0} & \mathbf{0} & \mathbf{0} & \mathbf{0} & \mathbf{0} & \mathbf{0} & \mathbf{C}_{5 \mathbf{a}}^{\mathbf{T}} & \mathbf{0} & \mathbf{0} \\
\mathbf{0} & \mathbf{0} & \mathbf{0} & \mathbf{0} & \mathbf{0} & \mathbf{0} & \mathbf{C}_{3 b}^{\mathbf{T}} & \mathbf{0} & \mathbf{0} & \mathbf{C}_{4 b}^{\mathbf{T}} & \mathbf{0} & \mathbf{0} & \mathbf{C}_{5 b}^{\mathbf{T}} & \mathbf{0} & \mathbf{0}
\end{array}\right]^{\mathbf{T}}
$$

The $\boldsymbol{\Omega}$ and $\boldsymbol{\Theta}$ in (16) are the generalized pressure vector and displacement vector given as $\boldsymbol{\Theta}=\left[\mathbf{a}_{1}, \mathbf{b}_{1}, \mathbf{c}_{1}, \mathbf{a}_{2}, \mathbf{b}_{2}, \mathbf{c}_{2}, \ldots \mathbf{a}_{5}, \mathbf{b}_{5}, \mathbf{c}_{5}\right]^{T}$ and $\boldsymbol{\Omega}=\left[\mathbf{e}^{\mathbf{a}}, \mathbf{e}^{\mathbf{b}}\right]^{T}$, respectively. The particular expression of coefficient matrix for $\mathbf{a}_{\mathbf{i}}, \mathbf{b}_{\mathbf{i}}, \mathbf{c}_{\mathbf{i}}$ and $\mathbf{e}^{\mathrm{I}}$ are given as shown.

$$
\begin{aligned}
& \mathbf{a}_{\mathbf{i}}=\left\{A_{i, 0,0}, A_{i, 0,1}, \ldots A_{i, m, 0}, A_{i, m, 1}, \ldots A_{i, m, n}, \ldots, A_{i, M, N}\right\}^{T} \\
& \mathbf{b}_{\mathbf{i}}=\left\{B_{i, 0,0}, B_{i, 0,1}, \ldots B_{i, m, 0}, B_{i, m, 1}, \ldots B_{i, m, n}, \ldots, B_{i, M, N}\right\}^{T} \\
& \mathbf{c}_{\mathbf{i}}=\left\{C_{i, 0,0}, C_{i, 0,1}, \ldots C_{i, m, 0}, C_{i, m, 1}, \ldots C_{i, m, n}, \ldots, C_{i, M, N}\right\}^{T} \\
& \mathbf{e}^{\mathbf{I}=}\left[E_{0,0,0}^{I}, E_{0,0,1}^{I}, \ldots, E_{0,0, M Z}^{I}, E_{0,1,0}^{I}, \ldots, E_{0,1, M Z}^{I}, \ldots, E_{0, M Y, M Z}^{I}, \ldots, E_{M X, M Y, M Z}^{I}\right]^{T}
\end{aligned}
$$

The Chebyshev polynomial series is truncated to $m x=M x, m y=M y, m \varepsilon=M E$, and $m=M$, $n=N$. The pressure inside the cavities and the flexible displacement of the plates can be derived in (12) to (15). For example, the free vibration problem for the structure-acoustic coupled system can be solved by assuming harmonic motion and removing the external load $\mathbf{F}$ in (16). The response of sound pressure and external load can be obtained by solving (16) and backsubstituting corresponding coefficients into the expression for the sound pressure and plate displacements. 


\section{Results and discussions}

The proposed models were used to study the vibration and sound transmission between two cavities separated by the I-shaped junction in the cascaded rectangular plate-cavity system. First, the results obtained by the proposed model were compared with the finite element (FE) method. Section 3.2 will illustrate the different boundary conditions and coupling that affects the vibroacoustic behavior of the coupled system. A parametric analysis was performed to gain knowledge on the general behavior of the structural-acoustic model of the cascaded rectangular plate-cavity system. The effect of cavity volume on the structure-borne sound transmission is investigated. The frequency range used in this study is between 0 to $500 \mathrm{~Hz}$. Nevertheless, a similar approach can be applied to other frequency range.

\subsection{Model validation}

The proposed model is suitable for both mechanical and acoustic excitation. Numerical examples and comparisons would be presented to validate the proposed method. In the proposed model, each acoustic domain has three rectangular surfaces in contact with the structure and other rigid surfaces. The dimensions of the source cavity $a$ and the receiver cavity $b$ are as follows: $L_{x, a}=0.4 m, L_{y, a}=04 m, L_{z, a}=02 m$, and $L_{x, b}=0.4 m, L_{y, b}=04 m, L_{z, b}=02 m$. A point source $(F=100 N)$ is applied to the plate $\mathrm{P} 1$ of cavity $a$ at the point $\left(x_{1}=0.2, y_{1}=0.2\right)$. The air density and the speed of sound are $\rho_{\text {air }}=1.21 \mathrm{~kg} / \mathrm{m}^{3}$ and $c_{0}=340 \mathrm{~m} / \mathrm{s}$, respectively. For simplification, all plates have the same material properties, namely: Young's modulus, $E=71 \mathrm{GPa}$, Poisson's ratio, $v=0.3$, the density, $\rho_{s}=2700 \mathrm{~kg} / \mathrm{m}^{3}$ and the plate thickness, $h=0.004 \mathrm{~m}$. The remaining sidewalls around the cavities are assumed to be perfectly rigid. In the proposed solution procedure, the rigid connection and clamp condition can be realized by setting all relevant stiffness values to a large number (in the order of $10^{12}$ ).

The accuracy of the proposed model is validated for mechanical excitation. The results obtained from the dynamic responses of the proposed method is compared with the finite element (FE) program named Hyperwork ${ }^{\mathrm{TM}}$. The validation finite element model was built using a $10 \mathrm{~mm} \times 10 \mathrm{~mm}$ cquad4 elements and chexa elements for the plate and acoustic components, respectively. In this study, the Chebyshev polynomial series is truncated: $M=N=14$ for the plate displacements and $M x=M y=M z=8$ for the cavity pressure. The computed dynamic responses are compared for both the proposed model and the FE model. Plate P2 and Plate P3 are far from the source plate in the source cavity and near to the source plate in the receiver cavity, respectively. They are chosen to verify their transverse vibration velocity. Figure 2(a) shows the transverse velocity response $\dot{w}$ of plate $\mathrm{P} 2\left(x_{2}=0.2, y_{2}=0.2\right)$ and $\mathrm{P} 3\left(x_{3}=0.2, y_{3}=0.2\right)$ in logarithmic scale. Figure 2(b) presents the sound pressure $p$ in the cavity a and cavity $b$ at the point $\left(X_{a}=0.2, Y_{a}=0.2, Z_{a}=0.1\right)$ and $\left(X_{b}=0.2, Y_{b}=0.2, Z_{b}=0.1\right)$, respectively. The reference values in the $\mathrm{dB}$ scales are Pa for sound pressure. It can be seen that there are some shift in natural frequency and slight discrepancies on resonant peaks. Nevertheless, both the vibrational and acoustic responses obtained by the proposed model exhibits a close match with the FE method. 

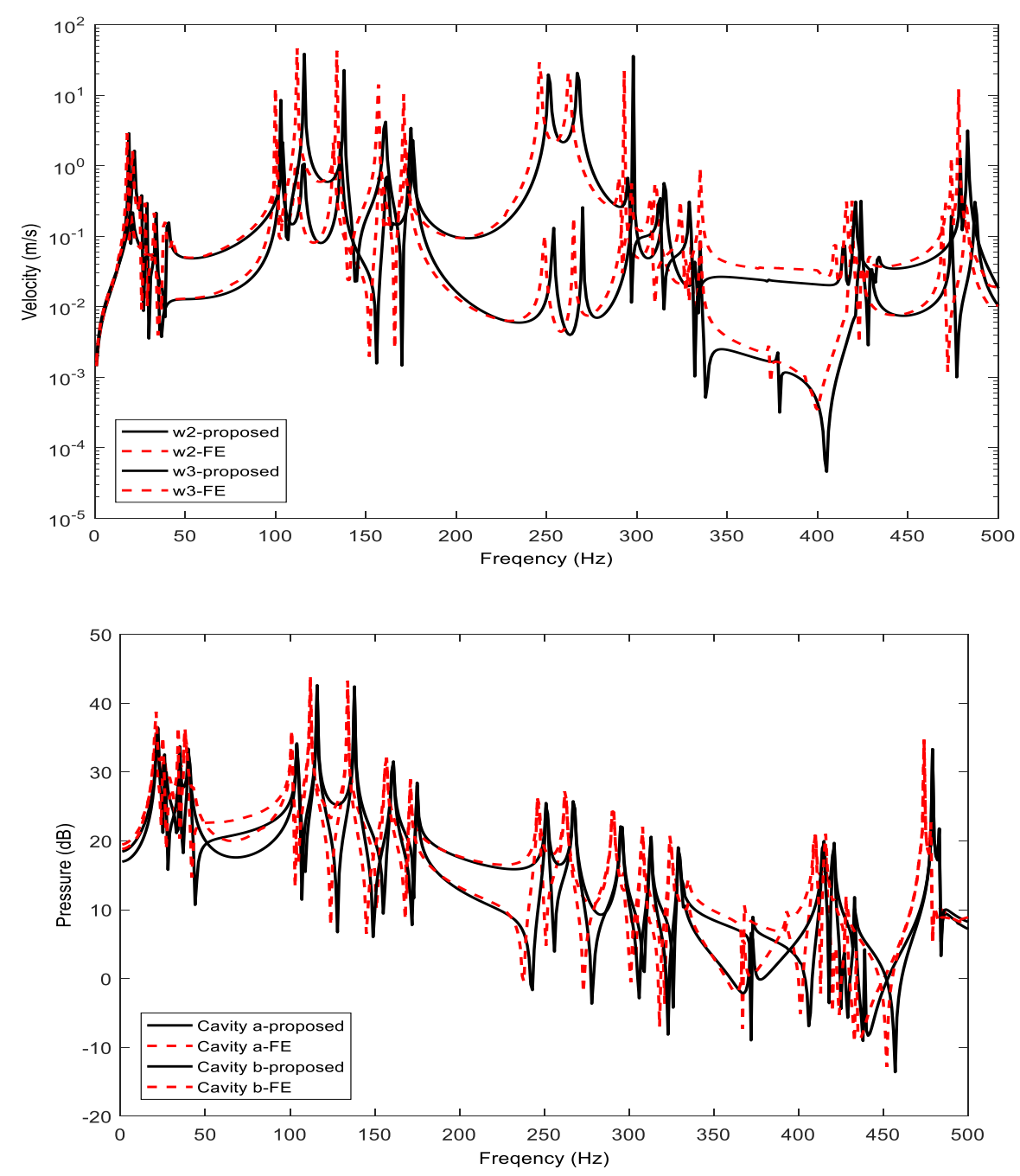

Figure 2 Model verification on (a) transverse velocity at the center of Plate P2 and P3; (b) pressure response in the cavity $a$ and $b$

\subsection{Parametric studies}

Several system parameters are used to investigate the structure-borne transmission characteristics on the acoustic response in the source and receiving cavities of the cascaded rectangular plate-cavity system. They are namely: boundary conditions, structural coupling manner, plate properties, and the volume ratio of source-to-receiver cavity.

\subsubsection{Effect of structural coupling manner and boundary condition}

From the viewpoint of structural vibration, the coupling manner and boundary condition can significantly affect the modal properties of the structural domain. However, it is not clear the mechanism of structural coupling and boundary conditions that affect the acoustic response in the source and receiving cavities. The investigation can be conducted using the proposed method. As mentioned in the previous section, the spring technique employed in the proposed method enables different boundary conditions and coupling described by changing the relevant spring stiffness values. 
In this case study, the structural dimensions and material properties of the coupled system are as follows. The dimension of the two cavities is $L x \times L y \times L z=0.4 m \times 0.4 m \times 0.2 m$. The density of air is $\rho_{\text {air }}=1.21 \mathrm{~kg} / \mathrm{m}^{3}$; the speed of sound in air is $c_{0}=340 \mathrm{~m} / \mathrm{s}$. The panels are made of steel with Young/s modulus $E=210 \mathrm{GPa}$, Poisson's ratio $v=0.3$, density $\rho_{s}=7850 \mathrm{~kg} / \mathrm{m}^{3}$, and thickness $h=0.004 \mathrm{~m}$. The bottom panel of the cavity a, P1, is excited by a point force $F=100 \mathrm{~N}$. Other sides of the cavities are perfectly rigid. The Chebyshev polynomial series is truncated to $M=N=14$ for the plate displacements and $M x=M y=M z=8$ the cavity pressure. The influences of structural coupling manner on the pressure response in the source cavity and receiving cavity are studied using different boundary conditions of each plate as tabulated in Table 1 .

Table 1: Boundary conditions used for coupled and disconnected case

\begin{tabular}{|c|c|c|c|c|c|}
\hline Cases & P1 & P2 & P3 & P4 & P5 \\
\hline Coupled & $x_{1}=0$ & - & $x_{3}=0$ & - & - \\
\hline Disconnected & $x_{1}=0,0.4$ & $x_{2}=0,0.4$ & $x_{3}=0,0.4$ & $x_{4}=0,0.4$ & $y_{5}=0,0.4$ \\
\hline
\end{tabular}

The pressure response generated by connecting and disconnected panels are quite different in both the source cavity $a$ and cavity $b$ (see Fig. 3). There are large variance on the amplitudes and modal properties. For example, in the source room, the numbers of resonant peaks are largely reduced, and the pressure response amplitudes are reduced as compared to the plate-coupled case. It implies that the coupled structural results in a higher pressure response in the source cavity. A more significant impact can be found in the receiver cavity. As shown in Fig.3(b), the only transmission path from Plate 5 leads to a low-pressure response in the receiver cavity after the vibration transmission was eliminated. The structure-borne noise in the source adjacent rooms (with thin-metal) due to the rigid structural coupling enables the vibration energy to be transmitted efficiently with minimal losses. The plate-cavity model may not be suitable for analyzing the vibro-acoustic behavior in the offshore platform. From noise control perspective, it is important to isolate the cavity from all vibrating structures to reduce the structure-borne noise radiation.

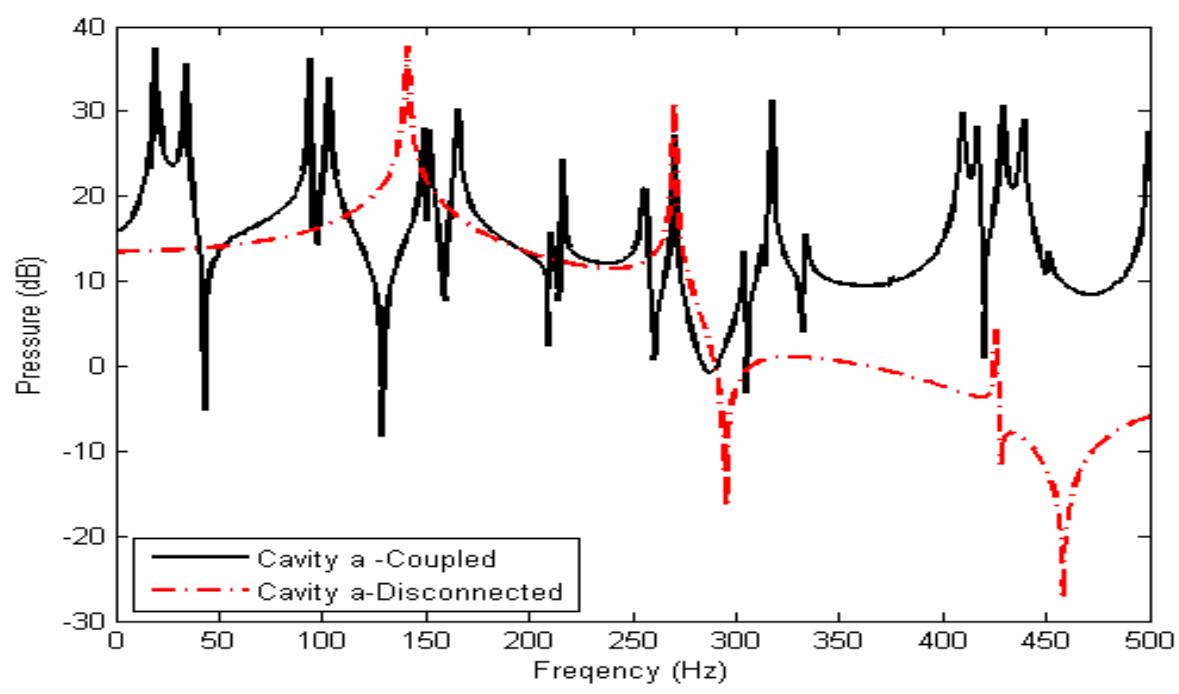




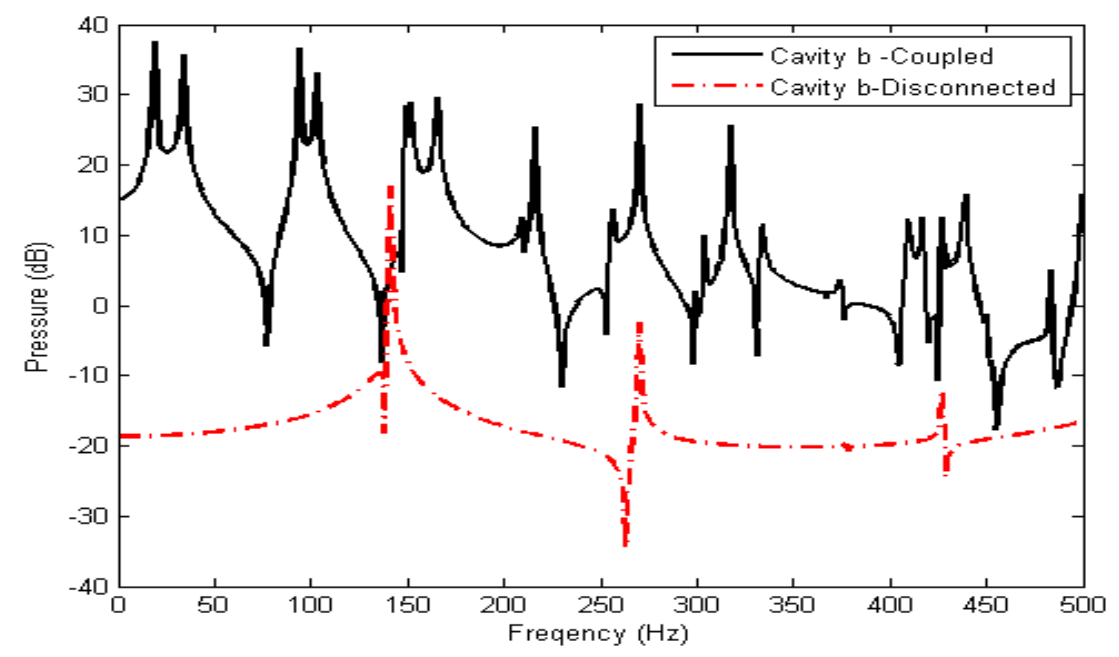

Figure 3 Influence of coupling manner on pressure response in (a) source cavity $a$; (b) receiver cavity $b$

Apart from the coupling manner between plates, the influences of boundary condition are also examined. The Plate P1 and P3 are assumed to be uniformly supported along the edge $x_{1}=0$ and $x_{3}=0$. The effect of the boundary spring stiffness on the natural frequencies can be seen in Fig. 4. As the translational and rotational restraining spring stiffness increased from zero (free) to infinity (clamped condition), the low restraining stiffness value $\left(0\right.$ and $\left.1 \times 10^{2}\right)$ has a small impact on the system natural frequencies. The intermediate stiffness values $\left(1 \times 10^{4}\right.$ and $\left.1 \times 10^{6}\right)$ have a strong influence on the system natural frequencies. However; as the restraining stiffness value increased to sufficient high value $\left(1 \times 10^{8}\right.$ and $\left.1 \times 10^{10}\right)$, the air cavity becomes a dominating factor affecting the natural frequency.

The increased in the restraint stiffness give a different result on cavity pressure responses to the modal behavior. Figure 5 shows both the source and receiving cavities exhibit a very large amplitude difference when the stiffness values are low $\left(0\right.$ and $\left.1 \times 10^{2}\right)$. The increased in the restraining stiffness reduced the response differences at low frequencies. It can be seen in the pressure responses in both cavities remain unchanged at restraint stiffness of sufficiently high value $\left(1 \times 10^{8}\right.$ and $\left.1 \times 10^{10}\right)$. 


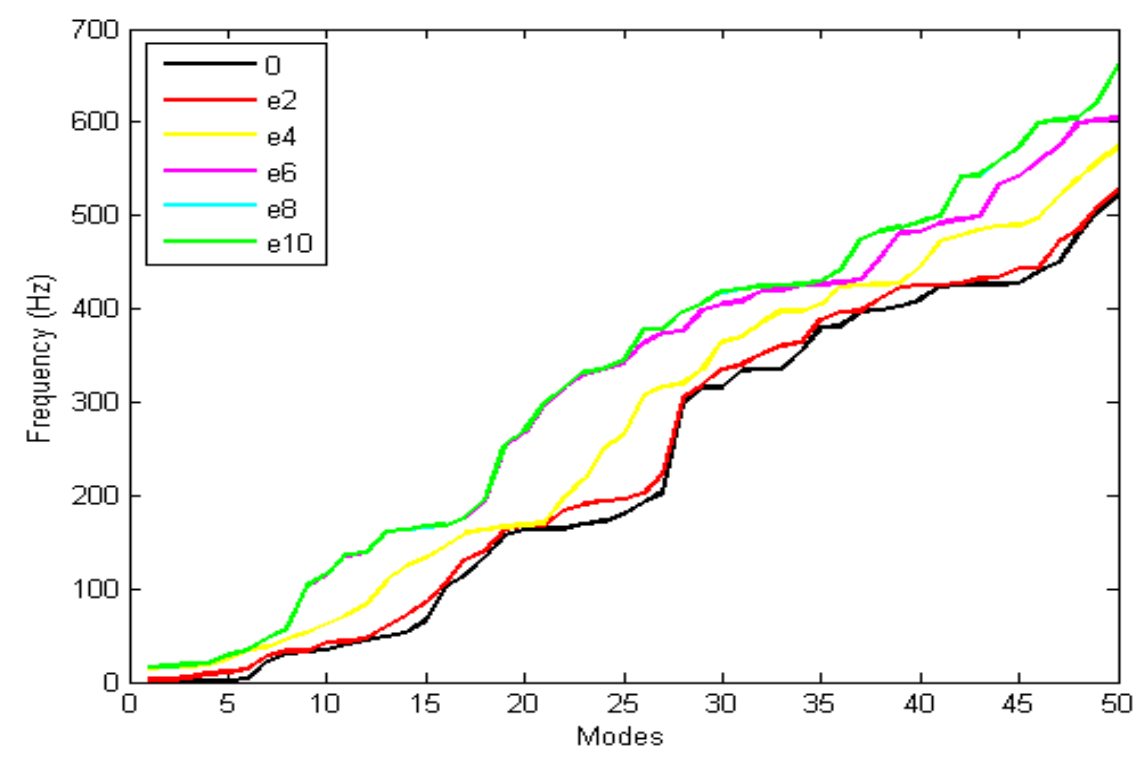

Figure 4 Effect of boundary spring stiffness on natural frequencies

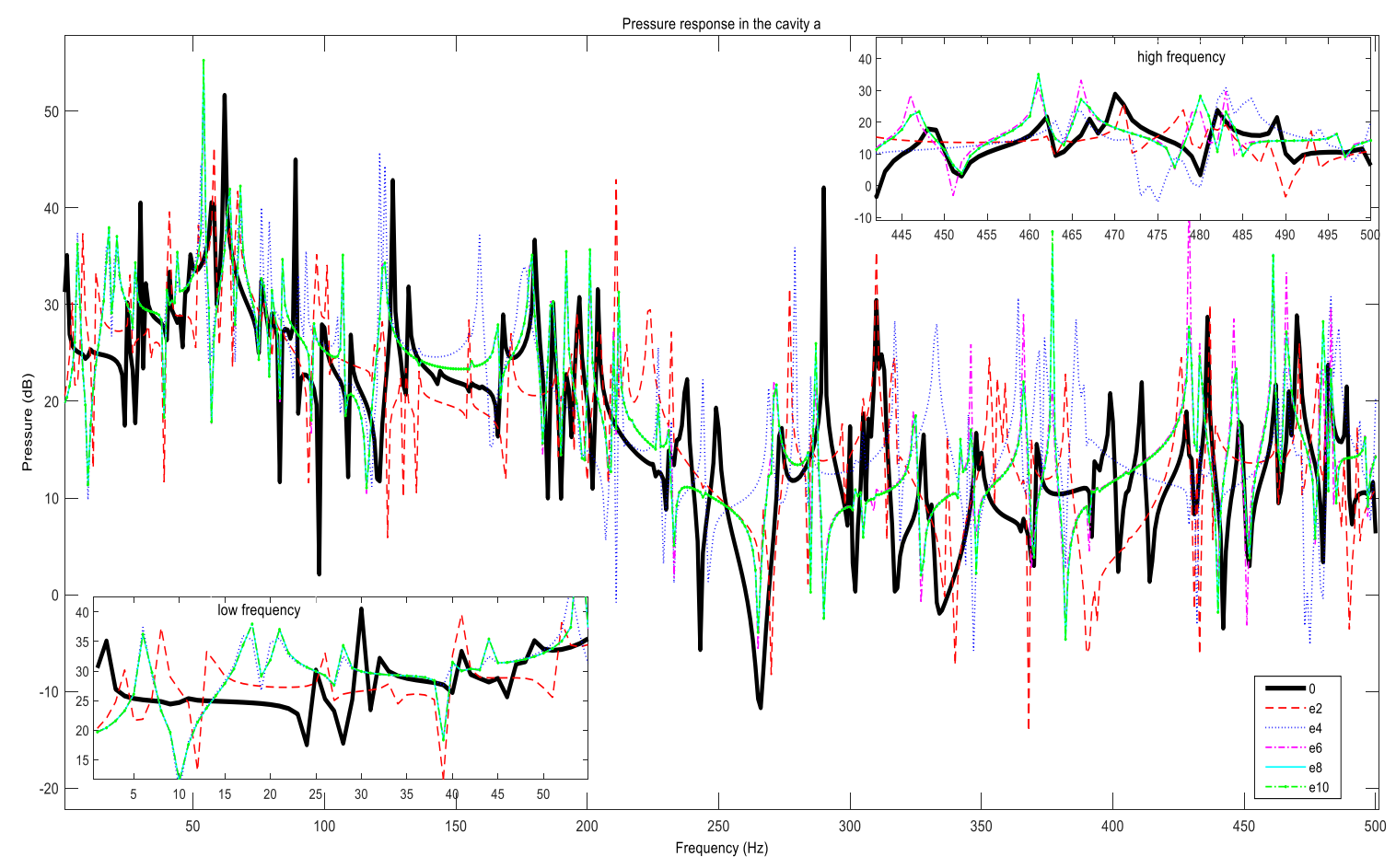




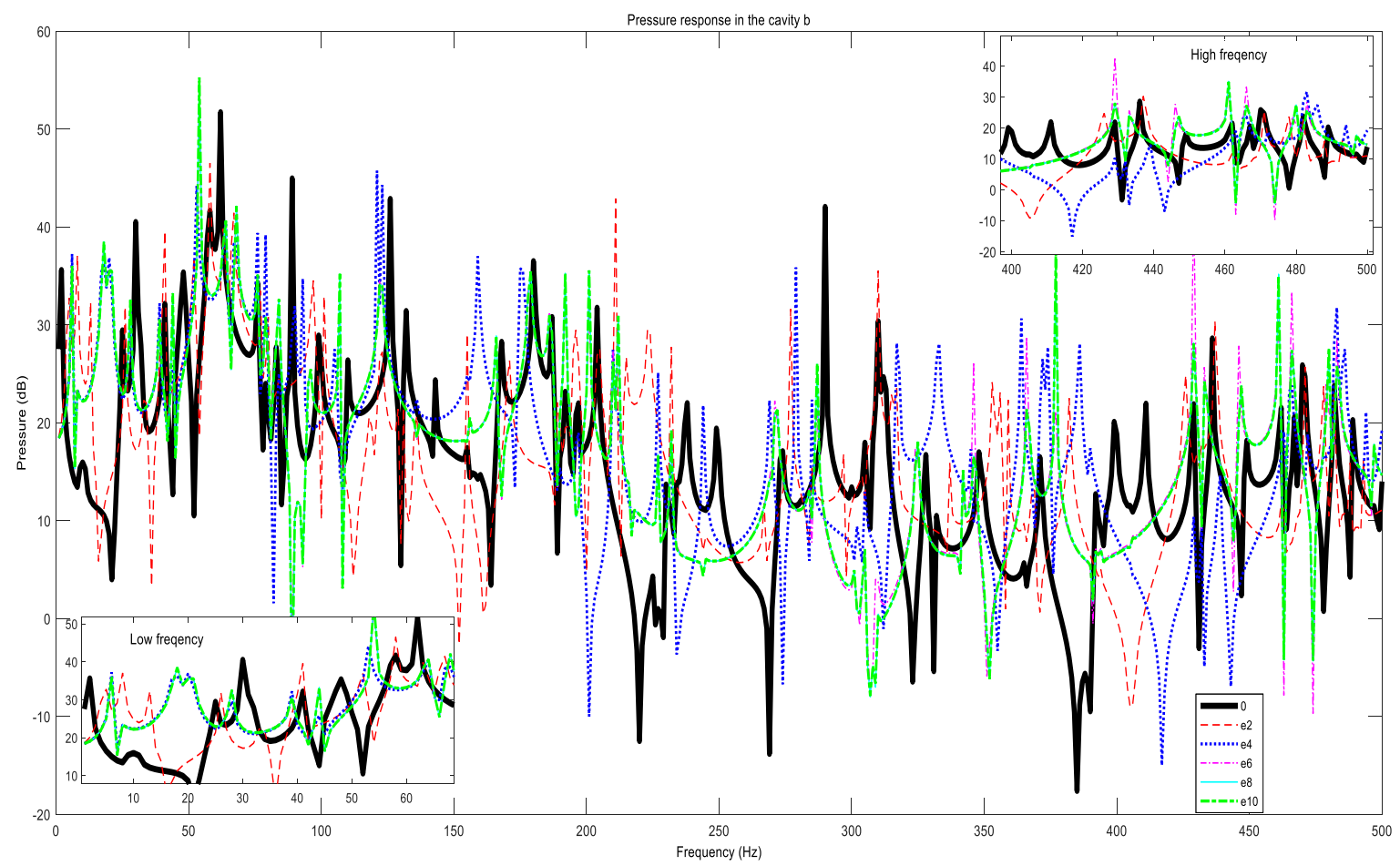

Figure 5 Effect of boundary stiffness on cavity pressure response on (a) source cavity $a$; (b) cavity $b$

\subsubsection{Effect of structure-property on the structure-borne sound transmission}

The structure-borne sound transmitted to the adjacent rooms is affected mainly by the physical properties of the structural domain. A parametric study on the structural domain could help to understand the inherent transmission mechanism. With the proposed model, the parametric study can be performed. In this case, plate P1 and P3 are assumed to be uniformly clamped along the edge $x_{1}=0$ and $x_{3}=0$ where all the plates are rigidly connected. The dimension of the two cavities remained as $L y=0.4 \mathrm{~m}, L z=0.2 \mathrm{~m}$. For simplicity, all the panels are assigned with identical material properties. Table 2 specifies two types of material properties attributed to the structural domain. Note that no damping or absorptive material is applied to both cavities.

Table 2 Material properties for parametric studies

\begin{tabular}{lllll}
\hline Material & $\rho_{s}\left[\mathrm{~kg} / \mathrm{m}^{3}\right]$ & $E[\mathrm{GPa}]$ & $h[\mathrm{~mm}]$ & $v[-]$ \\
\hline Steel & 7850 & 210 & $4,8,12$ & 0.3 \\
Aluminium & 2700 & 71 & $4,8,12$ & 0.3 \\
\hline
\end{tabular}

The pressure response in the source room is primarily influenced by the source plate P1. By calculating the vibrating velocity $\dot{w}$ of the source plate for different parameter change, the structural mobility can be expressed as 


$$
Y=\frac{\dot{w}}{F}
$$

Figure 6 shows the source plate's mobility where the plate thickness and the corresponding sound pressure response are obtained in the source room. It can be observed that a thin plate results in higher mobility and more substantial sound pressure response in the source room. Based on the obtained mobility spectrum in Figure 7, the aluminum plate generated higher mobility and sound pressure response in the source cavity for the same thickness. The mobility of the source plate is proportional to the acoustic pressure response due to the point force excitation. Both the cavity-controlled and panelcontrolled resonant peaks are excited in the acoustic pressure response curves. The resonant peaks at the panel-controlled frequencies are formed in the mobility curve. This phenomenon may be attributed to the weak coupling between the panel and air in the source cavity. It can be concluded that the source cavity acoustic pressure response is governed by the mobility of the source plate. Therefore, to reduce the structure-borne sound in a source room, one approach is to reduce the source plate's vibration velocity and to increase its impedance.
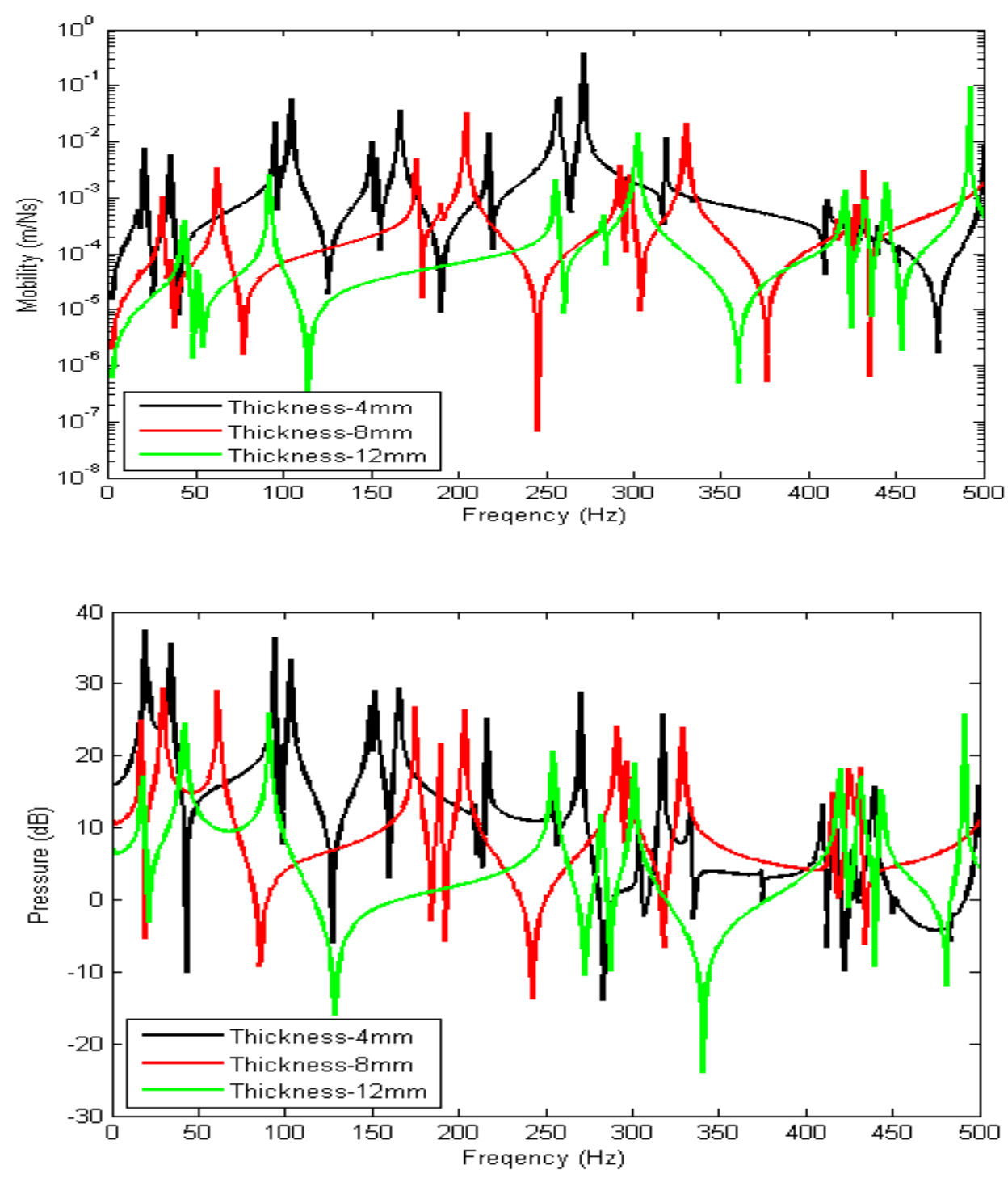

Figure 6 Effect of plate thickness on (a) mobility of source plate P1; (b) source cavity pressure response 

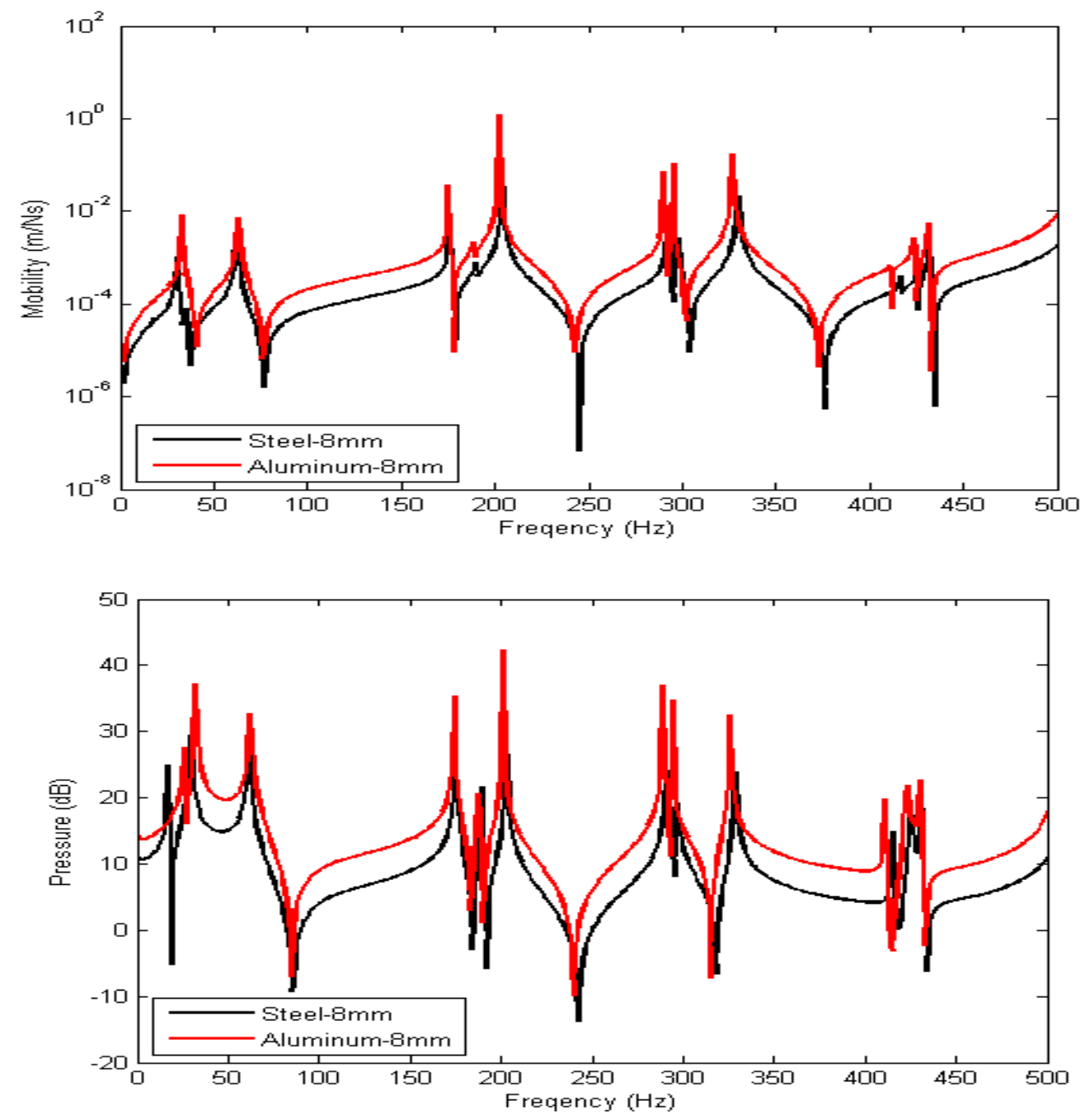

Figure 7 Effect of plate material on (a) mobility of source plate P1; (b) source cavity pressure response

As the plate thickness varies, the acoustic pressure responses in the receiver cavity behave differently than the source cavity. As observed in Fig. 8, the cavity enclosed by thinner plates resulted in a higher pressure response. As the plate thickness increased, the resonant peaks reduced. It can be deduced that the acoustic pressure response in the receiver cavity is mostly influenced by the resonance of the structural domain within the considered frequency range. On the other hand, Fig. 9 illustrates the situation when different materials were applied to the enclosed plates of identical thickness. A shift in the system resonant frequencies at the higher frequencies can be observed. The aluminum plate results in a higher resonance peak and pressure response amplitude as compared to the steel plate. It can be concluded that the receiver cavity acoustic response behaved similarly in the source cavity when the plate material and thickness changes. 


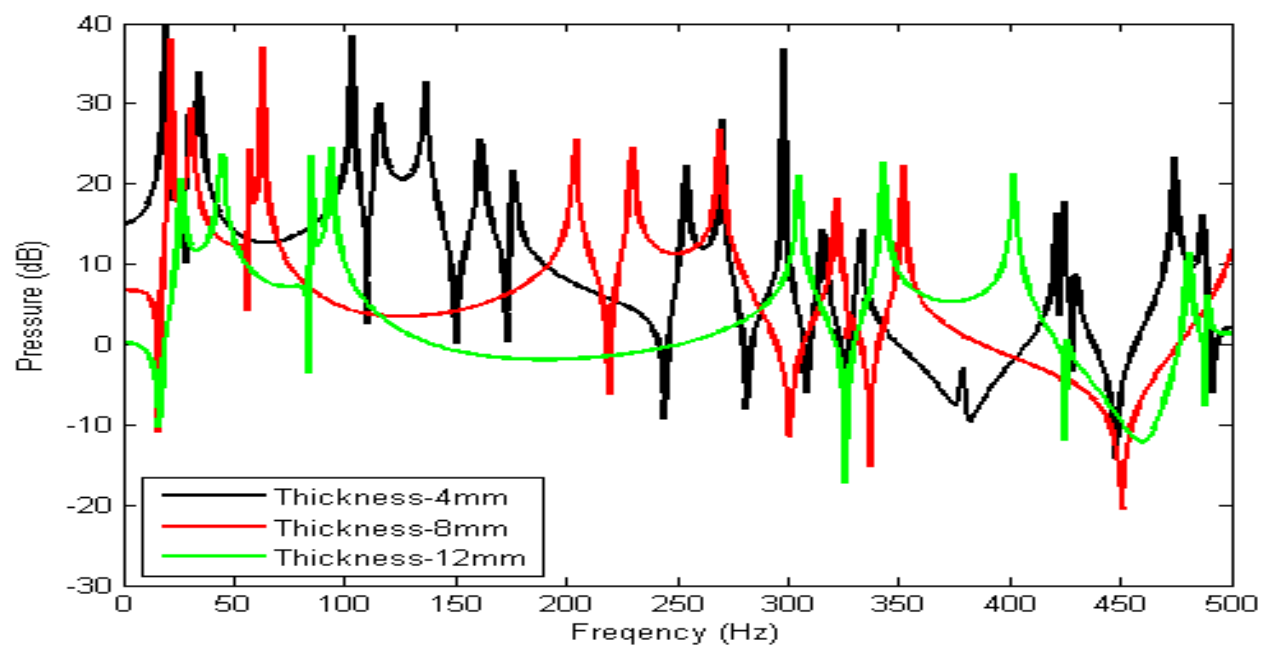

Figure 8 Effect of plate thickness on pressure response for receiver cavity $b$

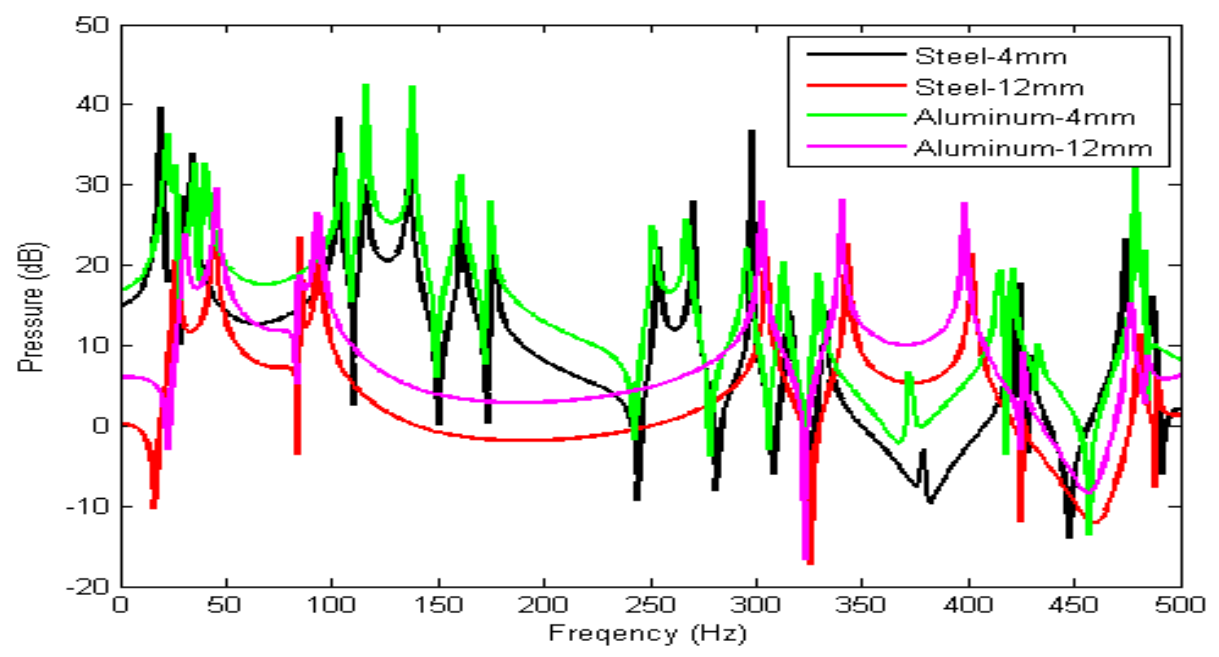

Figure 9 Effect of plate material on the pressure response of receiver cavity $b$

\subsubsection{Effect of cavity volume on the structure-borne sound transmission}

The types of configuration that increase the structure-borne sound's transmission were identified. In this study, three scenarios were considered: a larger source cavity, cavities of equal-size, and larger receiver cavity. The width and height of the two cavities are kept constant at $L y=0.4 \mathrm{~m}$ and $L z=0.2 \mathrm{~m}$, respectively. The position of the center wall will vary along the $x$ axis to create differences in the cavity depth. Subsequently, the volume ratios of the cavity a and cavity $b$ is $3: 1\left(L x_{a}=0.6 \mathrm{~m}, L x_{b}=0.2 \mathrm{~m}\right)$. The density of air is $\rho_{\text {air }}=1.21 \mathrm{~kg} / \mathrm{m}^{3}$; the speed of sound in air is $c_{0}=340 \mathrm{~m} / \mathrm{s}$. The panels are made of steel with Young/s modulus $E=210 \mathrm{GPa}$, Poisson's ratio $v=0.3$, density $\rho_{s}=7850 \mathrm{~kg} / \mathrm{m}^{3}$, and thickness $h=0.008 \mathrm{~m}$. For each volume ratio, a point force $F=100 N$ is acting at the bottom panel of the cavity a (named P1).

Figure 10 shows the source and receiver cavity acoustic pressure varies across different volume ratio. The higher positive value of $p_{a}-p_{b}$ implies a lower acoustic pressure response in the receiver cavity. It also shown that the configuration is less efficient on transmitting the structure- 
borne sound. Conversely, a higher negative value of $p_{a}-p_{b}$ implies that the acoustic pressure response in the receiver cavity is greater than the source cavity. Note that a spectrum with dense positive resonant peaks belonged to a larger source cavity. On the other hands, a spectrum with dense negative resonant peaks has a larger receiver cavity. As shown in Fig. 9, it can be concluded that the configuration with equal size source and receiver cavities is less effective for structure-borne sound transmission. However, a small source cavity with large receiver cavity generates higher negative $p_{a}-p_{b}$ value. But it provides a better configuration for structure-borne sound transmission among the considered scenarios.

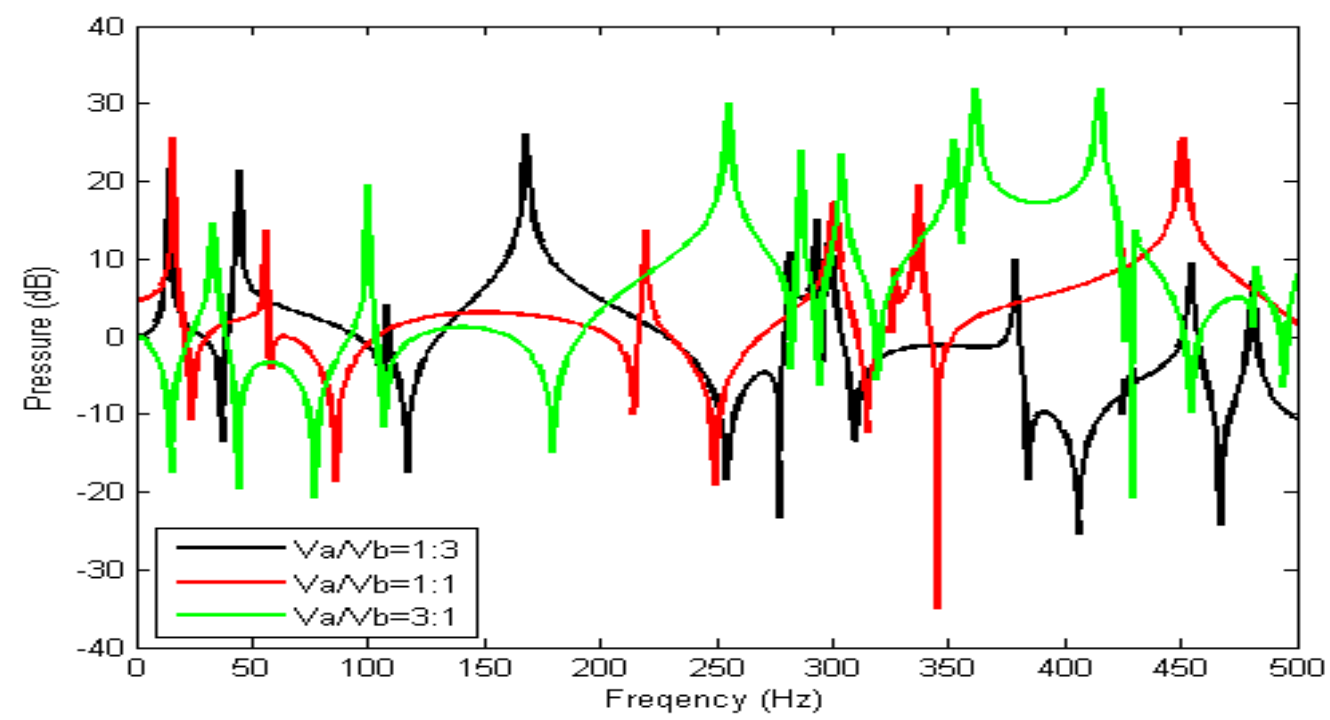

Figure 10 Comparison of source/receiver cavity acoustic pressure response difference 


\section{Conclusion}

This paper studied the structure-borne sound transmission through I-junction in the structural-acoustic model of a cascaded rectangular plate-cavity system using Chebyshev-Ritz method. Three-dimensional plate displacements and the sound pressure expression were described by double and triplicate Chebyshev series. The structure-structure coupling effects were included by considering the energy loss due to the boundary force and moments. The unknown coefficients of the displacements and sound pressure expression were obtained by performing the Ritz procedure. The displacement and pressure function was constructed by Chebyshev polynomial series of the first kind that was sufficiently smooth to ensure the derivative of the function exist and continuous at any point of the considered region. The Ritz solution is mathematically equivalent to solving of the governing differential equations and boundary coupling conditions.

The responses of the proposed model exhibited a close match with the finite element method despite the differences in the natural frequency and resonant peaks. Several system parameters such as boundary conditions, structural coupling manner, plate properties, and the volume ratio of the source-to-receiver cavity were used to investigate the structure-borne transmission characteristics on the acoustic response in the cavities.

The coupled structural produced higher pressure response in the receiver cavity than the source cavity. It is important to isolate the cavity from all vibrating structures to reduce the structure-borne noise radiation. As the translational and rotational restraining spring stiffness increased, the low restraining stiffness value has a smaller effect on the system natural frequencies than the intermediate stiffness values. The increased in the restraint stiffness gave different results on the cavity pressure response. Large amplitude variation was observed in both the source and receiving cavities at lower stiffness values. The increased in the spring stiffness reduced the response at a lower frequency. But the pressure responses in both cavities remain unchanged at higher stiffness value.

The effective way to reduce the source plate's vibration velocity and increase its impedance was reducing the structure-borne sound in the source room. The cavity enclosed by thinner plates resulted in a higher pressure response. The resonant peaks reduced as the plate thickness increased. The aluminum plate resulted in a higher resonance peak and pressure response amplitude as compared to the steel plate. The configuration with equal size source and receiver cavities was less effective for structure-borne sound transmission than small source cavity with large receiver cavity. Nevertheless, it produced better configuration for structure-borne sound transmission among the considered scenarios. 
The influence of these parameters on the structure-borne sound transmission of the configuration could be examined. The results can help engineers to understand the energy transmission mechanisms under varying parameter values and to provide a systematic approach for engineering noise control in a structural-acoustic model of the cascaded rectangular plate-cavity system such as the offshore platform with many rooms. A similar approach can be applied to other structural-acoustic system, and it can be extended to more than two structural-acoustic compartments. For future works, the proposed model will be further optimized and extended to inclined walls on more compartments will be performed. Machine learning techniques will be applied to predict the structure-borne sound transmission in the compartments.

\section{References}

[1] M. Schanz, Wave propagation in viscoelastic and poroelastic continua, New York: Springer, 2011.

[2] Keranen J, Hongisto V. "Comparison of simple room acoustic models used for industrial spaces," Acta Acustica United with Acustica. vol. 96, pp. 179-194, 2010.

[3] J.-H. Song, S.-Y. Hong, Y. Kang, H.-G. Kil, "Vibrational energy flow analysis of penetration beamplate coupled structures," Journal of Mechanical Science and Technology, vol. 25, no. 3, pp. 567-576, 2011.

[4] R. Craik and R. Smith, "Sound transmission through double leaf lightweight partitions part I: Airborne sound," Applied Acoustics, vol. 61, pp. 223-245, 2000.

[5] K. S. Sum and J. Pan, "A study of the medium frequency response of sound field in a panel-cavity system,” J. Acoust. Soc. Am, vol. 103, pp. 1510-1519, 1998.

[6] J. T. Du, W. L. Li, H. A. Xu and Z. G. Liu, "Vibro-acoustic analysis of a rectangular cavity bounded by a flexible panel with elastically restrained edges," J. Acoust. Soc. Am, vol. 131, no. 4, pp. 27992810, 2012.

[7] Y. H. Chen, G. Jin, S. Shi and Z. G. Liu, "A general analytical method for vibroacoustic analysis of an arbitrarily restrained rectangular plate backed by a cavity with general wall impedance," Journal of Sound and Vibration, vol. 136, pp. 031015-1-11, 2014.

[8] M. Pirnat, G. Cepon and M. Boltezar, "Structural-acoustic model of a rectangular plate-cavity system with an attached distributed mass and internal sound source: Theory and Experiment," Journal of Sound and Vibration, vol. 333, no. 7, pp. 2003-2018, 2014.

[9] K. Namcheol, "Aeroelastic Flutter Mechanisms of a Flexible Disk Rotating in an Enclosed Compressible Fluid," Journal of Applied Mechanics, vol. 71, pp. 120-130, 2004.

[10] J. Kyeong-Hoon and K. Heung-Seok, "Free vibration of multiple rectangular plates coupled with liquid,” International Journal of Mechanical Sciences, vol. 74, pp. 161-172, 2013.

[11] S. Arash and R. R. Ahmad, "Effects of in-plane loads on free vibration of symmetrically cross-ply laminated plates resting on Pasternak foundation and coupled with fluid," Ocean Engineering, vol. 115, pp. 196-209, 2016.

[12] X. Xie, H. Zheng and Y. g. Qu, "A variational formulation for vibro-acoustic analysis of a panel backed by an irregularly-bounded cavity," Journal of Sound and Vibration, vol. 373, pp. 146-163, 2016. 
[13] M. C. Remillieux, S. M. Pasareanu and U. P. Svensson, "Numerical modeling of the exterior-tointerior transmission of impulsive sound through three-dimensional thin-wall elastic structures," Journal of sound and vibration, vol. 332, pp. 6725-6742, 2013.

[14] S. X. Shi, G. Y. Jin and Z. G. Liu, "Vibro-acoustic behavior of an elastically restrained doublepanel structure with an acoustic cavity of arbitrary boundary impedance," Applied Acoustics, vol. 76, pp. 431-444, 2014.

[15] H. Seyyed M, K. Hojat A and S. Razgar, "Free vibration and dynamic response of a fluid-coupled double elliptical plat system using Mathieu function," International Journal of Mechanical Sciences, vol. 75, pp. 66-79, 2013.

[16] J. Nitin K and S. Venkata R, "Structural Acoustics of a Rectangular Panel Backed by a Cavity: An Analytical Matrix Approach," Journal of Vibration and Acoustics, vol. 139, pp. 031004-1-8, 2017.

[17] Y. Chen, G. Jin, Z. Feng and Z. Liu, "Modeling and vibro-acoustic analysis of elastically restrained panel backed by irregular sound space," Journal of Sound and Vibration, vol. 409, pp. 201-216, 2017.

[18] F. Fahy and P. Gardonio, Sound and structural vibration: radiation, transmission and response, Academic Press, 2007.

[19] A. Dijckmansa, G. Vermeir and W. Lauriks, "Sound transmission through finite lightweight multilayered structures with thin air layers," J. Acoust. Soc. Am., vol. 128, pp. 3513-3524, 2010.

[20] D. Desmet, “A wave based prediction technique for coupled vibro-acoustic analysis," K.U. Leuven, Division PMA, Leuven, 2008.

[21] Rayleigh, John William Strutt, Baron, The Theory of Sound, London, Macmillan, 1877.

[22] R. Walter, "Theorie der transversalschwingungen einer quadratischen platte mit freien randern," Annalen Der Physik, vol. 333, no. 4, pp. 737-786, 1909.

[23] J. T. Du, G. y. Jin, T. j. Yang and Z. g. Liu, "An analytical method for the in-plane vibration analysis of rectangular plates with elastically restrained edges," Journal of sound and vibration, vol. 306, pp. 908-927, 2007.

[24] J. T. Du, W. L. Li, Z. g. Liu, T. j. Yang and G. y. Jin, "Free vibration of two elastically coupled rectangular plates with uniform elastic boundary restraints," Journal of sound and vibration, vol. 330, pp. 788-804, 2011.

[25] J. Lee, "Application of Chebyshev-tau method to the free vibration analysis of stepped beams," International Journal of Mechanical Sciences, Vols. 101-102, pp. 411-420, 2015.

[26] D. Zhou, S. Lo and Y. Cheung, "3-D vibration analysis of annular sector plates using the Chebyshev-Ritz method," Journal of Sound and Vibration, vol. 320, pp. 421-437, 2009.

[27] M. Filippi, A. Pagani, M. Petrolo, G. Colonna and E. Carrera, "Static and free vibration analysis of laminated beams by refined theory based on Chebyshev polynomials," Composite Structures, vol. 132, pp. 1248-1259, 2015.

[28] Y. Chen, G. Jin and Z. Liu, "Flexural and in-plane vibration analysis of elastically restrained thin rectangular plate with cutout using Chebyshev-Lagrangian method," International Journal of Mechanical Sciences, vol. 89, pp. 264-278, 2014. 
[29] Y. Xiao, D. Shao, H. Zhang, C. Shuai, Q. Wang, "An acoustic modeling of the three-dimensional annular segment cavity with various impedance boundary conditions," Results in Physics, vol.10, pp. 411-423, 2018.

[30] Y. Sun, T. Yang, Y. Chen, "Sound radiation modes of cylindrical surfaces and their application to vibro-acoustics analysis of cylindrical shells," Journal of Sound and Vibration, vol. 424, no. 23, pp. 64$77,2018$.

[31] U. Orrenius, H. Liu, A. Wareing, S.Finnveden, V.Cotoni, "Wave modelling in predictive vibroacoustics: Applications to rail vehicles and aircraft, Wave Motion," vol.51, no. 4, pp. 635-649, 2014.

[32]G. Y. Jin, Y. H. Chen and Z. G. Liu, "A Chebyshev-Lagrangian method for acoustic analysis of rectangular cavity with arbitrary impedance walls," Applied Acoustics, vol. 78, pp. 33-42, 2014.

[33] Y. Chen, G. Jin, Z. Feng and Z. Liu, "Modeling and vibro-acoustic analysis of elastically restrained panel backed by irregular sound space," Journal of Sound and Vibration, vol. 409, pp. 201-216, 2017.

[34] X. Xie, H. Zheng and Y. G. Qu, "A variational formulation for vibro-acoustic analysis of a panel backed by an irregularly-bounded cavity," Journal of Sound and Vibration, vol. 373, pp. 146-163, 2016.

[35] H. Zhang, D. Shi, S. Zha, Q.Wang, "Parameterization study on the moderately thick laminated rectangular plate-cavity coupling system with uniform or non-uniform boundary conditions," Composite Structures, vol. 194, pp. 537-554, 2018.

[36] J. T. Du, Y. Liu, Y. Wang, G. Wang, "Vibro-acoustic analysis of an elastically restrained plate duct silencer backed by irregular acoustical cavity," Applied Acoustics, vol. 138, pp. 60-71, 2018.

[37] H. Zhang, D. Shi, Q. Wang, S.Zha, "Vibro-acoustic analysis of the annular segment flexible plate coupled with an impedance walled enclosure," Thin-Walled Structures, vol. 131, pp. 205-222, 2018.

[38] S. Shi, Z. Su, G. Jin, Z. Liu, "Vibro-acoustic modeling and analysis of a coupled acoustic system comprising a partially opened cavity coupled with a flexible plate," Mechanical Systems and Signal Processing, vol. 98, pp. 324-343, 2018. 